\title{
SOME CONSEQUENCES OF CRITICAL REFRACTION \\ IN THE VENUS ATMOSPHERE
}

\author{
CONWAY W. SNYDER \\ Jet Propulsion Laboratory, Pasadena, Calif., U.S.A.
}

\begin{abstract}
Mariner V and the three successful Venera probes have shown the density of the atmosphere of Venus to be so high that critical refraction occurs at an altitude near $35 \mathrm{~km}$. Somewhat bizarre optical effects are to be expected in such an atmosphere. Using a spherically symmetric model based upon the measurements of the refractivity at the top of the atmosphere by the Mariner V S-band occultation experiment, the Venera 4 data in its altitude range, and adiabatic extrapolation to the surface, calculations of a variety of ray paths have been made with a double-precision computer program. Phenomena discussed include the magnification of the planet, the elevation of the horizon, the apparent motion and distortion of the sun, and the possibility of 'seeing' completely around the planet.
\end{abstract}

Both the Mariner $\mathrm{V}$ mission and the Venera 4 mission demonstrate conclusively the existence of the phenomenon of critical refraction in the Venus atmosphere. This means that the atmosphere is so dense that there is a level at which a horizontal ray of electromagnetic radiation would have a curvature equal to the distance from that level to the center of the planet and hence it would be bent into a circle and returned to its original point. Horizontal rays above this level would be less refracted and would not meet the surface of the planet if extended in either direction, whereas horizontal rays below the critical level would encounter the planet's surface at both ends. Since the somewhat bizarre effects that can be produced by critical refraction has been commented upon rather inaccurately in both the Soviet and American press, it seems appropriate to consider the matter quantitatively so as to put the phenomena in the proper perspective.

For these calculations, I have used a model of the Venus atmosphere which is based upon the measurements of the refractivity at the top of the atmosphere by the Mariner V S-band occultation experiment and fits smoothly on to the Venera 4 model down to the level at which the probe ceased communicating. Using Kliore's latest range scale, this occurred at $6078 \mathrm{~km}$. Since the Venera 4 data indicates a near adiabatic lapse rate at this level, I have extrapolated on down along an adiabat to the range of $6050 \mathrm{~km}$ corresponding to the radar value for the radius of the planet. At this level, the model gives a temperature close to $770 \mathrm{~K}$, a pressure of $135 \mathrm{~atm}$, and a density 78 times as great as the earth's atmosphere at the surface. The atmosphere is assumed to consist practically entirely of $\mathrm{CO}_{2}$ and the refractive index corresponding to S-band microwaves has been used.

The critical level in this atmosphere occurs at $6087.8 \mathrm{~km}$. Since this is probably 20 to $25 \mathrm{~km}$ below the top of the opaque clouds, certain of the interesting phenomena that are possible will not actually occur with visible light, although they may occur in the microwave region where the transparency of the atmosphere is higher. For this

Sagan et al. (eds.), Planetary Atmospheres, 36-38. All Rights Reseried. Copyright 1971 by the I.A.L. 
discussion, I shall simply imagine that the atmosphere is infinitely transparent and examine what phenomena we might see.

As we approach the planet, the first thing we discover is that the apparent radius of the planet is $6099.5 \mathrm{~km}$. This is a sort of magnification effect of the atmosphere and is independent of the actual size of the planet. It comes about because a ray which approaches the planet in a direction to pass by it a distance slightly larger than 6099.5 $\mathrm{km}$ from the center will be refracted inward, become tangential just outside the critical level, and pass back out through the atmosphere again. However, a ray that is very slightly closer to the planet will be refracted inward, will spend a very long time near the critical level, but will never quite become tangential, and will then spiral in and strike the surface. The apparent size of the planet is independent of our distance from the planet so long as we are outside the atmosphere, but as soon as we have a little of the atmosphere behind us, the magnification changes. Thus when we actually reach the range of $6099.5 \mathrm{~km}$ where the surface had appeared to be, we will find that the surface is now at $6096 \mathrm{~km}$. When we reach $6096 \mathrm{~km}$ we will find it to be at $6094 \mathrm{~km}$, and when we reach $6094 \mathrm{~km}$, the planet will still be slightly smaller than $6092 \mathrm{~km}$. Somewhere along here the planet's apparent size begins to increase, since when we get to the limiting altitude the planet looks infinitely large. This is because we can see the surface by looking horizontally along the critical ray in any direction or more exactly by looking an infinitesimal angle below the horizontal. The planet in fact appears to be a very flat bowl with its edges extremely far away and just at the level where we are sitting.

Let us consider for a moment the outermost grazing rays that can actually penetrate the atmosphere from outside and reach the surface. It reaches the 19 bar level at $6078 \mathrm{~km}$ with a slope of $1.97^{\circ}$. If the surface is actually $28 \mathrm{~km}$ lower down, as I suspect, the ray then continues downward going another $3.4^{\circ}$ around the planet before reaching $6050 \mathrm{~km}$ which it approaches with a slope of $9.4^{\circ}$. It has been stated that it would appear to an observer on Venus that he was always at the bottom of a hole. For the 19 bar atmosphere, a more apt description would be at the center of a very flat saucer. If he looks outward along a ray that is more than about $10^{\circ}$ upward, the amount of bending in that ray is almost negligible. Even at a $4^{\circ}$ elevation the effect is small and looking upward $2^{\circ}$ he can still see out into space.

If the planet radius is $6050 \mathrm{~km}$ then the effect is very much larger. Looking upward at an elevation angle of $45^{\circ}$ the ray is deflected downward by more than $1^{\circ}$. At $30^{\circ}$ it is deflected by more than $2^{\circ}$ and at $10^{\circ}$ it is deflected downward by more than $12^{\circ}$. Thus our flat saucer becomes more like a bowl. The theoretical horizon which is thousands of $\mathrm{km}$ away is elevated by an angle of $9.3976^{\circ}$.

For example, I have calculated two rays at this angle which differ in elevation at the observer by 3 ten-millionths of a degree. Fifty degrees around the planet from the observer, the lower ray is just returning to the surface, while the upper ray is already $200 \mathrm{~km}$ above the surface and is rising rapidly. Any opacity in the atmosphere would lower the visible horizon quite appreciably. For example, if a point $200 \mathrm{~km}$ away is the farthest we could see then the horizon would appear elevated by only $5^{\circ}$.

A particularly interesting set of phenomena accompany the motion of the sun. 
Here again we must imagine that we are able to see in wavelengths of electromagnetic radiation to which the entire atmosphere is transparent. Suppose that we are standing on the equator at the time of the equinox so that the sun is directly overhead at noon. First of all, the sun travels from west to east because of the retrograde rotation of the planet. Second, the sun moves very slowly, only $3^{\circ}$ per twenty-four hour day, but then Venusians are accustomed to much longer days. Around noon we see nothing unusual; even at 3:00 pm when we know the sun is $45^{\circ}$ above the horizontal it appears to be at $46.2^{\circ}$. But along about 5:00 pm the sun begins to move very much more slowly and changes its shape instead. In fact, its vertical dimension gets smaller and smaller at just the same rate that its velocity of descent decreases. At 6:00 pm when it should be along the horizontal, it is still up $10.4^{\circ}$. After all, the horizon is up $9.4^{\circ}$ Its distortion is now severe; its horizontal dimension is approximately normal but its vertical dimension has been reduced by a factor of 4 . By 7:00 pm this vertical reduction has reached 250 times and by 8:00 pm it has reached 30000 times. As midnight approaches its vertical dimension continues to get smaller and smaller. At some time - it is very difficult to calculate just when - the horizontal dimension of the little sliver that is the sun begins to increase very rapidly and just at midnight it reaches all the way around, defining by a very narrow bright line the distant horizon. Then the line breaks in the east, becomes shorter and shorter and the sun gradually reassembles itself at the western horizon, gradually regaining its normal circularity as it rises. Of course if we happen to be situated more than about $\frac{3}{8}$ of a degree off of the latitude of the sun, we will not be treated to the sight of the uniformly illuminated midnight horizon. Instead, the little sliver of the sun will simply crawl like a worm across the horizon during the night from the point where it has set to the point where it is planning to rise. 